\title{
CONTROLE QUÍMICO DA CERCOSPORIOSE DO MILHO NA SAFRINHA
}

\author{
Gisèle Maria Fantin ${ }^{1}$ \\ Aildson Pereira Duarte ${ }^{2}$ \\ Renato Arantes Pinto ${ }^{3}$
}

\section{RESUMO}

A cercosporiose do milho é uma das doenças que mais reduzem a produtividade desta cultura em inúmeros países. No Brasil, era de ocorrência secundária, mas nos últimos anos, adquiriu importância, chegando a causar severas epidemias na safra 1999/2000 no Estado de Goiás. Como muitos cultivares ainda não apresentam resistência satisfatória à doença, torna-se necessário recorrer ao uso de produtos químicos para seu controle quando ocorrem condições favoráveis ao desenvolvimento de epidemias. O presente trabalho teve como objetivo estudar o efeito de fungicidas para o controle da cercosporiose do milho na safrinha. Os ensaios foram conduzidos em Palmital-SP e FloríneaSP, em 2002, com o híbrido duplo Balu 184, suscetível à doença. Os produtos, suas formulações e dosagens ( $\mathrm{mL}$ ou g i.a./ha) testados foram: Carbendazim SC (500), Fluquinconazole PM + óleo mineral CE $(250+187,5)$, Carbendazim + Fluquinconazole + óleo $(250+125+187,5)$, Carbendazim + Tebuconazole CE $(250+100)$, Trifloxystrobin GD + Tebuconazole $(75+100)$, comparados a dois fungicidas registrados para a cultura, Tebuconazole (200) e Pyraclostrobin + Epoxiconazole SE $(99,75+37,5)$ e a uma testemunha sem aplicação de produto. Foram usados 6 blocos casualizados com 6 repetições. Cada parcela consistiu de 4 linhas de $6 \mathrm{~m}$, sendo as duas centrais consideradas como parcela útil. Os produtos foram pulverizados no milho perto do florescimento,

\footnotetext{
${ }^{1}$ Instituto Biológico, Caixa Postal 70, CEP 13001-970, Campinas-SP. E-mail: gisele@biologico.sp.gov.br

${ }^{2}$ Apta Médio Paranapanema, Caixa Postal 263, CEP 19800-000, Assis-SP.

${ }^{3}$ Bayer Crop Science, Av. Maria Coelho Aguiar, 215, Bl. B, $2^{2}$ a., CEP 05804-902, São Paulo-SP.
} 
quando foi realizada uma avaliação prévia. A avaliação final foi feita em função do desenvolvimento da doença, após 27 dias em Palmital e 40 em Florínea. Todos os produtos causaram redução do número de lesões da cercosporiose, sendo mais eficientes Carbendazim + Fluquinconazole + óleo e Carbendazim seguidos por Carbendazim + Tebuconazole e Pyraclostrobin + Epoxiconazole. Foi observada também a ocorrência da mancha de Phaeosphaeria, para a qual o tratamento Trifloxystrobin + Tebuconazole apresentou controle muito semelhante ao do padrão Pyraclostrobin + Epoxiconazole. Não foi observado efeito dos tratamentos sobre a produtividade devido à baixa intensidade das doenças.

Palavras-chave: fungicidas, Mancha de Cercospora, Zea mays, Cercospora zeae-maydis, Cercospora sorghi var. maydis, Phaeosphaeria maydis.

\section{ABSTRACT}

\section{CHEMICAL CONTROL OF MAIZE GRAY LEAF SPOT IN THE SECOND SEASON}

Gray leaf spot is one of the most yield-limiting diseases of maize in many countries. In Brazil it progressively emerged during the last years and severe epidemics happened at the 1999/2000 crop in the State of Goiás. Since many cultivars were not yet improved to acceptable levels of resistance to the gray leaf spot, chemical management tactics should be necessary under favorable environmental conditions to the disease. The objective of this work was to compare fungicide treatments to the control of gray leaf spot at the second season maize. Field experiments were conducted at Palmital SP and Florínea-SP, in 2002, with the susceptible double hybrid Balu 184 . The products, formulations and rates ( $\mathrm{mL}$ or g a.i./ha) used were: Carbendazim SC (500), Fluquinconazole WP + mineral oil EC $(250+187,5)$, Carbendazim + Fluquinconazole + oil $(250+125+187,5)$, Carbendazim + Tebuconazole EC $(250+100)$, Trifloxystrobin WG + Tebuconazole $(75+100)$, compared with two registered fungicides for the culture: Tebuconazole (200) and 
Pyraclostrobin + Epoxiconazole SE $(99,75+37,5)$. A nontreated control was also included. The Experimental design was a randomized complete block with six replications. Each plot consisted of four rows with six meters. The two central rows were used for the assessments. The preliminary disease assessment and fungicides spray were carried out close to flowering of plants. The final evaluation was dependent on the disease development. It was carried out 27 and 40 days after the preliminary one in Palmital and Florínea, respectively. All products reduced the gray leaf spot lesions number. The most efficient were Carbendazim + Fluquinconazole + oil and Carbendazim followed by Carbendazim + Tebuconazole and Pyraclostrobin + Epoxiconazole. Phaeosphaeria leaf spot also occurred in the experiment. For this disease, the data obtained with the mixture Trifloxystrobin + Tebuconazole were close to the best results achieved by the registered control Pyraclostrobin + Epoxiconazole. The treatments did not increase yield due to the low disease condition.

Key words: fungicides, Zea mays, Cercospora zeae-maydis, Cercospora sorghi var. maydis, Phaeosphaeria maydis.

\section{INTRODUÇÃO}

A cercosporiose ou mancha de Cercospora é uma doença foliar de importância recente na cultura do milho no Brasil. Anteriormente considerada doença secundária, sua intensidade foi aumentando nos últimos anos e epidemias foram primeiramente relatadas na safra 1999/ 2000, no Estado de Goiás (Fernandes \& Oliveira, 2000). Esta doença disseminou-se rapidamente para outras regiões, sendo constatada, já no ano seguinte, em vários outros Estados do País, principalmente da região Centro-Sul (Fantin et al., 2001).

Os fungos agentes causais da doença são Cercospora zeaemaydis, encontrado na maioria das lesões e considerado o principal patógeno, e também $C$. sorghi var. maydis, encontrado com pequena freqüência. Os sintomas são lesões foliares estreitas e alongadas, com os bordos caracteristicamente limitados pelas nervuras secundárias. Estes patógenos sobrevivem principalmente em restos de cultura de milho 
chuva, podendo ser levados a longas distâncias pelo vento. Períodos prolongados de chuva, com elevada umidade relativa e temperatura entre 22 e $30^{\circ} \mathrm{C}$ favorecem o desenvolvimento da doença (White, 2000).

Doença mundialmente importante, a cercosporiose vem causando danos à produtividade do milho nos EUA há mais de 25 anos. A expansão da doença nesse país, tanto em distribuição geográfica como em intensidade, ocorreu concomitantemente com ao aumento, em extensas áreas das regiões produtoras de milho, das práticas de plantio direto. Apesar da intensificação, a partir da década de 1990, dos estudos visando ao aumento da resistência dos híbridos comerciais, considerado o método ideal de controle (Donahue et al., 1991; Carson et al., 2002), é comum a perda da resistência devido à variabilidade genética do patógeno (Dunkle $\&$ Carson, 1998). Desta forma, o uso de híbridos muito produtivos mas suscetíveis, associado à prática contínua do plantio direto sob monocultura, têm elevado cada vez mais o potencial de inóculo do patógeno nos restos culturais nas regiões produtoras de milho dos EUA. Importantes perdas na produção foram observadas repetidamente naquele país e a área de ocorrência da doença foi se expandindo a cada ano (Latterell \& Rossi, 1983; Lipps, 1998). Em muitas circunstâncias, portanto, principalmente em campos de produção de sementes, tem sido imprescindível o uso de fungicidas para evitar danos elevados à produtividade da cultura. Apenas as práticas de manejo em pequenas extensões não têm se mostrado efetivas quando há alto potencial de inóculo na região, devido à rápida disseminação do patógeno a longas distâncias pelo vento (Ward et al., 1999; Munkvold et al., 2001).

A cercosporiose é muito antiga e pandêmica também na África do Sul, onde há híbridos comerciais com níveis mais elevados de resistência que nos EUA e também existem linhagens com importantes fontes adicionais de resistência (Saghai Maroof et al., 1993; Gevers et al., 1994; Ward et al., 1999). Apesar disso, muitas vezes têm sido utilizados fungicidas para possibilitar a obtenção de um manejo econômico da doença (Asea et al., 2002).

Embora alguns fungicidas protetores, como o Chlorothalonil, proporcionem controle razoável da cercosporiose, produtos sistêmicos como benzimidazóis, triazóis e estrobilurinas têm se mostrado mais efetivos (Ward et al., 1999). 
Ward et al. (1997a), estudando o controle da cercosporiose em KwaZulu-Natal (África do Sul) concluíram que fungicidas do grupo dos triazóis em mistura com benzimidazóis ou com triazóis foram altamente efetivos. Os autores observaram que essas misturas resultaram em maior diminuição da doença, maior duração do controle e produtividade mais elevada. Observaram que as dosagens comerciais de Difenoconazole (triazol) e Carbendazim (benzimidazol), recomendadas naquele país, foram adequadas para o controle desta doença enquanto que as de Benomyl, Flutriafol e Flusilazole+Carbendazim foram insuficientes. Ressaltaram ainda a importância do uso de dois produtos em combinação na redução do risco de aparecimento de resistência do patógeno aos fungicidas. Em ensaios complementares, realizados também na África do Sul, na região de Pietermaritzburg, Ward et al. (1997b) estudaram freqüências e épocas de aplicação da combinação Carbendazim + Flusilazole e observaram respostas da produtividade aos fungicidas em função, principalmente, do estádio de desenvolvimento do hospedeiro quando do início das pulverizações, da quantidade de doença na época da pulverização, da duração do controle com o produto e de um controle efetivo durante a maturação fisiológica das plantas.

Nos EUA, Munkvold et al. (2001) obtiveram controle da doença tanto com Mancozeb, (produto de contato), como com Propiconazole (triazol comumente utilizado em campos de produção de sementes nesse país desde 1994). Estes autores relataram que uma única aplicação de Propiconazole foi, em geral, lucrativa em Iowa, onde a doença tem sido severa, mas ressaltaram que a probabilidade de controle vantajoso é altamente influenciada pela suscetibilidade do híbrido.

Ward et al. (1999) ponderam que a estratégia de manejo sustentável da mancha de Cercospora, a longo prazo, seja baseada no uso de cultivares altamente produtivos, resistentes e adaptados à região, de forma a reduzir a taxa de aumento da doença dentro da própria safra. Estes autores também consideram que esta estratégia seja mais facilmente obtida na África, onde há uma maior gama de materiais com níveis aceitáveis de resistência. Por outro lado, julgam ser mais difícil nos países desenvolvidos, onde o potencial produtivo é de extrema importância e o germoplasma mais suscetível. Para o controle da doença a curto prazo, devido à suscetihilidade dns cultivares avaliam nue deve ser feito um 
manejo integrado, podendo ser necessário o uso de fungicidas para reduzir a taxa de progresso da doença, associado à prática da rotação de culturas e manejo dos restos culturais, os quais minimizam grandemente o inóculo inicial. Outras medidas citadas pelos autores, e que podem complementar o manejo da doença, são densidade de plantas, irrigação e fertilidade do solo adequadas.

No Brasil, pela importância recente da doença, a resistência do germoplasma disponível e sua herança ainda são apenas parcialmente conhecidos; muitos híbridos e variedades de milho atualmente disponíveis para comercialização ainda não foram melhorados satisfatoriamente para resistência a esta doença, apresentando variados graus de resistência e suscetibilidade. Assim sendo, além da necessidade de complementar o controle da doença através de práticas culturais, principalmente a rotação de culturas, torna-se necessário, em condições ambientais favoráveis à doença, utilizar o controle químico. A importância do uso de fungicidas, além da diminuição da severidade da doença na lavoura, está na redução do inóculo nos restos da cultura que serão deixados no solo e da disseminação da doença para outras regiões.

Embora vários trabalhos venham sendo realizados sobre o controle químico desta doença do milho na região sudoeste de Goiás, ainda não há produtos registrados para seu controle no Brasil. Pinto et al. (2002) estudaram o efeito de 3 triazóis (Propiconazole, Difenoconazole e Tebuconazole) e uma Estrobilurina (Azoxystrobin) e concluíram que todos foram eficientes no controle da cercosporiose. Em seu estudo não foram incluídos benzimidazóis.

Por outro lado, Ramos et al. (2001a) e (2001b) e Brandão et al. (2001), estudando o efeito de fungicidas de contato (Ditiocarbamato, Mancozeb e óxido cuproso), benzimidazóis (Benomyl, Carbendazim e tiofanato-metílico), triazóis (Difenoconazole, Epoxiconazole, Fluquinconazole, Imibenconazole e Propiconazole), e uma estrobilurina (Azoxystrobin), isolados, em misturas ou associados a óleo, observaram maior redução da severidade da doença pelo uso da estrobilurina, embora nem sempre associada à maior produtividade.

Portanto, devido à importância da doença, à eventual necessidade de controle químico para evitar epidemias e à não existência de produtos morictradne no Rracil fni efetıadn n nresente trabalho. que teve como 
objetivo estudar o efeito, no controle da cercosporiose, de cinco tratamentos químicos comparados ao de dois fungicidas considerados padrões, já registrados para outras doenças na cultura do milho.

\section{MATERIAL E MÉTODOS}

Foram instalados cinco ensaios no Estado de São Paulo, nas regiões onde a mancha de Cercospora ocorreu com maior severidade no ano anterior (safrinha 2001), sendo dois em Guaíra e os demais em Ituverava, Palmital e Florínea. Entretanto, devido à seca generalizada que ocorreu durante o desenvolvimento dos experimentos, acarretando paralisação da doença, foram mantidos apenas os campos de Palmital e Florínea, nos quais as condições ambientais apresentavam-se mais favoráveis à evolução da doença, para dar continuidade aos ensaios (aplicação dos tratamentos).

O ensaio de Palmital foi semeado em 18/03/02 e o de Florínea em 29/03/02, ambos com espaçamento entre linhas de $0,8 \mathrm{~m}$. Foi utilizado o híbrido duplo Balu 184, suscetível à mancha de Cercospora. Realizouse adubação de semeadura de $500 \mathrm{~kg} / \mathrm{ha}$ da fórmula 05-25-25.

A avaliação prévia da intensidade da mancha de Cercospora e uma única aplicação dos fungicidas foram realizadas em 05/06/02, logo após o florescimento das plantas em Palmital, e no pré-florescimento em Florínea.

Avaliou-se a incidência da cercosporiose através da contagem do número de lesões na quarta folha abaixo da espiga principal (localizada no terço inferior da planta), em 20 plantas ao acaso no campo.

Foi quantificada ainda a mancha de Phaeosphaeria, outra doença de ocorrência natural nas plantas, para mensurar fontes de variação externas ao objetivo do ensaio. A avaliação foi feita, em 20 plantas ao acaso, na folha imediatamente abaixo da espiga (terço médio da planta), através da estimativa da severidade da doença, com auxílio de uma escala com ilustrações de seções de folhas de milho com porcentagens de área foliar afetada de 2, 5, 10, 15, 20 e 35\%, elaborada pelo Setor de Fitopatologia da ESALQ/USP. Em Florínea, foi também avaliada a incidência desta doença pela contagem do número de lesões na referida folha. 


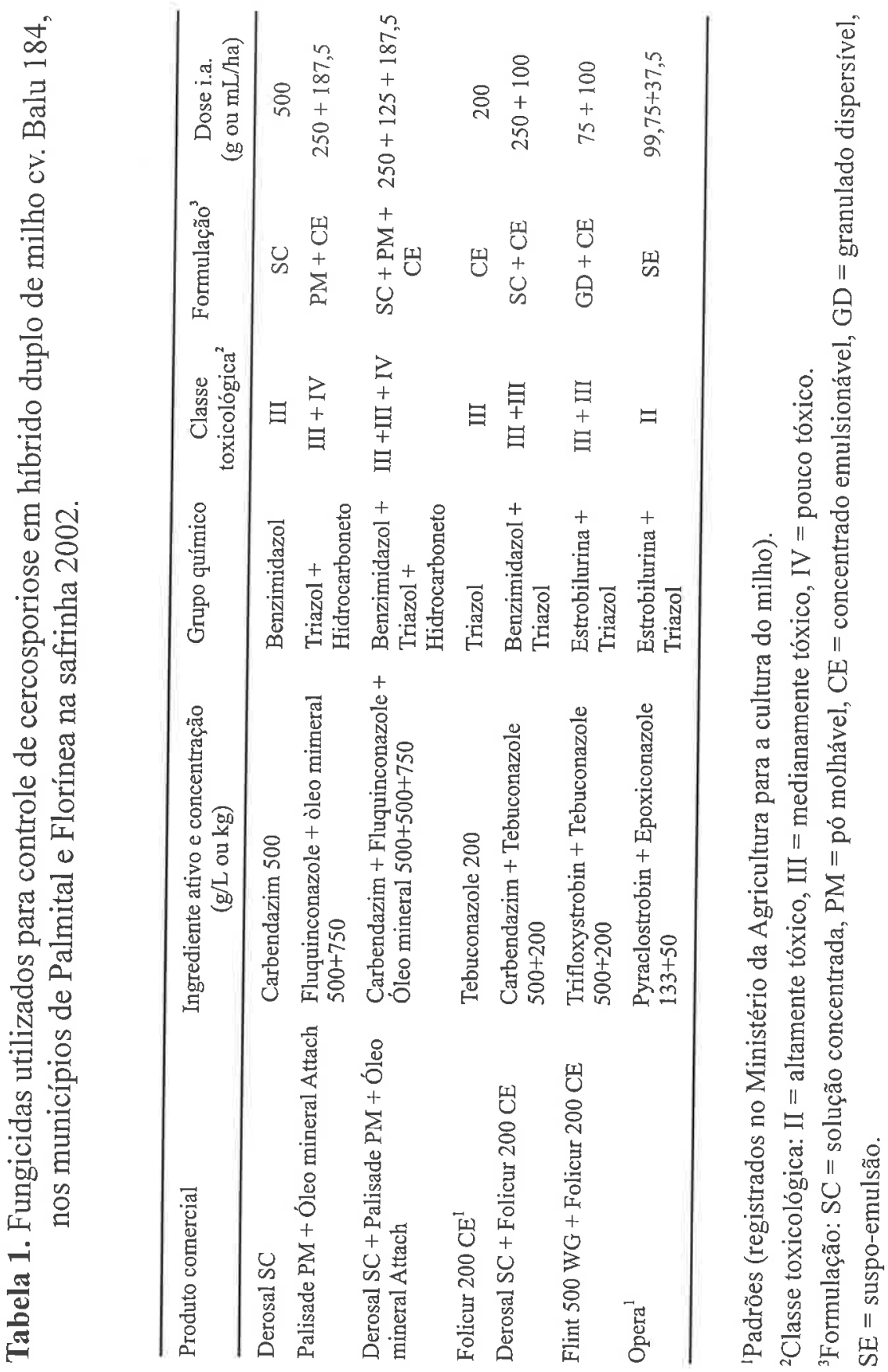


Após a avaliação das doenças, o campo foi demarcado, adotandose seis blocos casualizados com 8 tratamentos. As parcelas constituíramse de 4 linhas de $6 \mathrm{~m}$. Os tratamentos foram os constantes da Tabela 1.

Os produtos foram aplicados com pulverizador costal com barra, utilizando bicos 110 015, à pressão de 45 PSI e volume de $230 \mathrm{~L} / \mathrm{ha}$.

Em Palmital, a pulverização foi feita das $11 \mathrm{~h} 30$ às $12 \mathrm{~h} 20$, sob vento médio de aproximadamente 4 milhas $/ \mathrm{h}$, nebulosidade de $10 \%$, temperatura de $28,5^{\circ} \mathrm{C}$ e umidade relativa de $54 \%$. As plantas estavam secas no momento da aplicação.

Em Florínea, a pulverização deu-se das $16 \mathrm{~h} 15$ às $17 \mathrm{~h} 00$, com vento de 2 milhas/h, nebulosidade de 30 a $40 \%$, temperatura de $28^{\circ} \mathrm{C}$, umidade relativa de 48 a $54 \%$; as plantas também se encontravam secas.

A avaliação final de doenças em Palmital foi realizada em 02/ 07/02 e em Florínea em 15/07/02, através da contagem do número de lesões da cercosporiose na folha imediatamente abaixo da espiga principal de 10 plantas por parcela. Avaliou-se também a severidade da mancha de Phaeosphaeria, atribuindo-se notas de 1 a 9 para a parcela, correspondendo aos níveis de $0 ; 1 ; 2,5 ; 5 ; 10 ; 25 ; 50 ; 75$ e mais de $75 \%$ de área foliar afetada, com auxílio de uma escala diagramática elaborada pela Agroceres (Agroceres, 1993).

O ensaio de Palmital foi colhido em 19/08/02. Nessa localidade, foram obtidas as seguintes características agronômicas das plantas: altura de plantas e de espigas, índice de espigas, número de plantas acamadas e quebradas, população de plantas, rendimento de espigas, umidade dos grãos e massa de grãos. A produtividade foi obtida calculando-se a produção de cada parcela em quilos por hectare com base no peso de grãos, corrigida a umidade para $13 \%$.

Foi realizada análise de variância da intensidade das doenças e das características agronômicas. A comparação de médias de tratamentos foi feita através do teste de Tukey a 5\%. Para análise, os dados de (x) intensidade das doenças foram transformados em $\sqrt{x}$.

\section{RESULTADOS E DISCUSSÃO}

Houve bom desenvolvimento das plantas nos ensaios de Palmital e Florínea, porém a produtividade foi relativamente baixa. As doenças 
apresentaram baixa severidade, possivelmente por condições climáticas como pouca umidade e pequena quantidade de inóculo presente na região.

$\mathrm{Na}$ avaliação prévia à aplicação dos tratamentos, em 05/06/02, obteve-se incidência média de 2,85 e de 1,65 lesões da mancha de Cercospora na quarta folha abaixo da espiga das plantas em Palmital e em Florínea, respectivamente.

A mancha de Phaeosphaeria, avaliada na mesma data, apresentou severidade de $2,72 \%$ de área foliar afetada na folha imediatamente abaixo da espiga em Palmital e de $0,17 \%$ em Florínea. A incidência desta doença, obtida na mesma folha, em Florínea, , foi de 7,05 lesões.

Embora a precipitação pluvial do mês de maio na região (AssisSP) tenha sido de $177 \mathrm{~mm}$, após a aplicação dos fungicidas ocorreu um periodo de estiagem de 45 dias, desfavorecendo a evolução da doença.

Os dados de intensidade das doenças após a aplicação dos fungicidas, estão apresentados nas Tabelas 2 e 3 . Pela baixa intensidade de ambas as doenças, não deve ter havido influência de uma sobre o desenvolvimento da outra.

Apesar da incidência muito pequena da doença, foi possível observar que todos os produtos testados causaram redução do número de lesões da mancha de Cercospora. A eficiência de cada fungicida em relação aos demais foi semelhante em ambos os locais. Houve menor número de lesões com a aplicação de Carbendazim + Fluquinconazole + óleo mineral e Carbendazim seguidos por Carbendazim + Tebuconazole e Pyraclostrobin + Epoxiconazole.

Quanto à mancha de Phaeosphaeria, a aplicação de Trifloxystrobin + Tebuconazole proporcionou o controle que mais. se aproximou ao do padrão Pyraclostrobin + Epoxiconazole, registrado para o controle desta doença na cultura.

Em Florínea, a eficiência do tratamento químico foi melhor que em Palmital, havendo maior redução dos sintomas com a aplicação dos produtos (Tabelas 2 e 3 ). Isto pode ter sido causado pela pulverização das plantas um pouco mais jovens, ainda no pré-florescimento, com menor severidade inicial das doenças. A influência de vários fatores na eficiência do controle foi bastante discutida por Ward et al. (1997b).

Houve semelhança dos resultados aqui obtidos com os de Ward et al. (1997a e b) quanto à eficiência da mistura Benzimidazol + Triazol 
Tabela 2. Intensidade de doenças foliares no hibrido duplo de milho Balu 184 aos 106 dias após a semeadura, em Palmital - SP. Avaliação em 02/07/02, 27 dias após o tratamento químico.

\begin{tabular}{|c|c|c|}
\hline Tratamento & $\begin{array}{c}\text { Mancha de Cercospora } \\
\text { (incidencia }{ }^{2} \text { ) }\end{array}$ & $\begin{array}{c}\text { Mancha de } \\
\text { Phaeosphaeria } \\
\text { (severidade }{ }^{3} \text { ) }\end{array}$ \\
\hline $\begin{array}{l}\text { Carbendazim + Fluquinconazole + Óleo mineral } \\
\text { Carbendazim } \\
\text { Carbendazim + Tebuconazole } \\
\text { Pyraclostrobin + Epoxiconazole } \\
\text { Tebuconazole } \\
\text { Fluquinconazole + Oleo mineral } \\
\text { Trifloxystrobin + Tebuconazole } \\
\text { Testemunha } \\
\text { Média geral } \\
\text { CV }\end{array}$ & $\begin{array}{l}2,4 \mathrm{~d} \\
2,6 \mathrm{~d} \\
2,9 \mathrm{~cd} \\
3,4 \mathrm{~cd} \\
4,4 \text { bc } \\
5,2 \mathrm{~b} \\
5,3 \mathrm{~b} \\
8,4 \mathrm{a} \\
4,3 \\
11,1 \%\end{array}$ & $\begin{array}{l}1,76 \mathrm{ab} \\
1,92 \mathrm{ab} \\
1,91 \mathrm{ab} \\
1,18 \mathrm{c} \\
1,62 \mathrm{a}-\mathrm{c} \\
2,16 \mathrm{a} \\
1,53 \mathrm{bc} \\
1,93 \mathrm{ab} \\
1,76 \\
8,4 \%\end{array}$ \\
\hline
\end{tabular}

${ }^{1}$ Médias seguidas por letras distintas na mesma coluna diferem entre si ao nivel de $5 \%$ de significância pelo teste de Tukey. Análise dos dados ( $\mathrm{x}$ ) transformados em $\sqrt{x}$. ${ }^{2}$ Número de lesões na folha imediatamente abaixo da espiga. Média de 10 plantas/ parcela.

${ }^{3}$ Notas de 1 a 9 correspondendo, respectivamente, às porcentagens de área foliar afetada de $0 ; 1 ; 2,5 ; 5 ; 10 ; 25 ; 50 ; 75$ e mais de $75 \%$ da mancha de Phaeosphaeria na planta inteira

para o controle da cercosporiose. Munkvold et al. (2001) obtiveram controle lucrativo da doença pelo uso de um triazol, mas não estudaram o uso da mistura.

Comparando o presente trabalho com outros conduzidos recentemente no Estado de Goiás, sob elevada intensidade da doença, observa-se que os resultados obtidos estão de acordo com os de Pinto et al. (2002) que também obtiveram controle da cercosporiose com os triazóis e a estrobilurina testados, mas diferem dos de Ramos et al. (2001a) e (2001b) e Brandão et al. (2001), que encontraram melhor controle com o uso da Estrobilurina em comparação com o de triazóis e Benzimidazóis. Aventa-se a possibilidade destas variações resultarem do uso de ingredientes ativos diversos, embora do mesmo grupo químico, do uso de diferentes misturas de produtos com ou sem adição de óleo, de diferentes épocas de aplicação dos produtos, da variabilidade genética do patógeno e da variação na intensidade da doença e nas condições ambientais dos locais e épocas, entre outros fatores.

Quanto à produtividade da cultura, não foi possível observar 
Tabela 3. Intensidade de doenças foliares no hibrido duplo de milho Balu 184 aos 106 dias após a semeadura, em Florínea - SP. Avaliação em 15/07/02, 40 dias após o tratamento químico.

\begin{tabular}{|c|c|c|}
\hline \multirow[b]{2}{*}{ Tratamento } & \multicolumn{2}{|c|}{ Doenças' } \\
\hline & $\begin{array}{c}\text { Mancha de Cercospora } \\
\text { (incidência }{ }^{2} \text { ) }\end{array}$ & $\begin{array}{c}\text { Mancha de Phaeasphaeria } \\
\text { (severidade }{ }^{3} \text { ) }\end{array}$ \\
\hline Carbendazim + F luquinconazole + Óleo mineral & $0,28 \mathrm{c}$ & $1,68 \mathrm{ab}$ \\
\hline Carbendazim & $0,37 \mathrm{c}$ & $1,93 \mathrm{ab}$ \\
\hline Carbendazim + Tebuconazole & $0,50 \mathrm{c}$ & $1,62 \mathrm{ab}$ \\
\hline Pyraclostrobin + Epoxiconazole & $0,48 \mathrm{c}$ & $1,16 \mathrm{~b}$ \\
\hline Tebuconazole & $0,78 \mathrm{bc}$ & $1,53 \mathrm{ab}$ \\
\hline Trifloxystrobin + Tebuconazole & 0,85 bc & $1,10 \mathrm{~b}$ \\
\hline Fluquinconazole + Óleo mineral & $1,72 \mathrm{~b}$ & $2,00 \mathrm{ab}$ \\
\hline Testemunha & {$[4,00$ a } & 2,17 a \\
\hline Média geral & 2,37 & 1,65 \\
\hline $\mathrm{CV}$ & $22,1 \%$ & $15,8 \%$ \\
\hline
\end{tabular}

'Médias seguidas por letras distintas na mesma coluna diferem entre si ao nível de 5\% de significância pelo teste de Tukey. Análise dos dados (x) transformados em $\sqrt{x}$. ${ }^{2}$ Número de lesões na folha imediatamente abaixo da espiga. Média de 10 plantas/ parcela.

${ }^{3}$ Notas de 1 a 9 correspondendo, respectivamente, às porcentagens de área foliar afetada de $0 ; 1 ; 2,5 ; 5 ; 10 ; 25 ; 50 ; 75$ e mais de $75 \%$ da mancha de Phaeosphaeria na planta inteira

diferenças entre os tratamentos, como já era esperado, pela pequena intensidade dos sintomas nas plantas (Tabela 4). Ward et al. (1996), Ward et al. (1997b) e Munkvold et al. (2001) também observaram menor efeito dos fungicidas na produtividade sob baixa severidade da doença.

\section{CONCLUSÕES}

Todos os produtos testados apresentaram controle da mancha de Cercospora, destacando-se Carbendazim + Fluquinconazole + óleo mineral e Carbendazim seguidos por Carbendazim + Tebuconazole e Pyraclostrobin + Epoxiconazole. Não foi possível observar influência dos tratamentos na produtividade, por ter sido baixa a severidade da doença. 


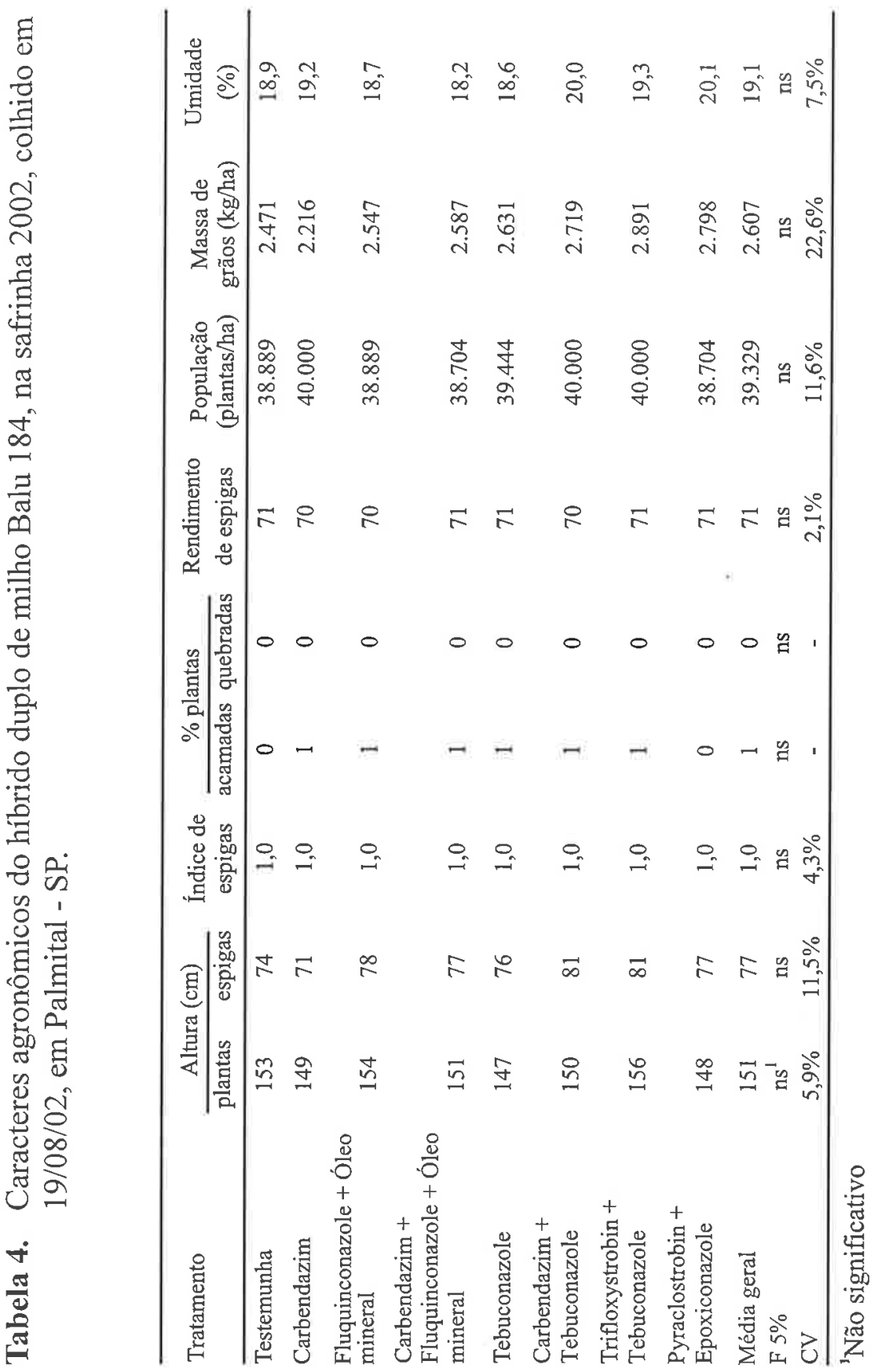




\section{REFERÊNCIAS BIBLIOGRÁFICAS}

AGROCERES, 1993. Guia Agroceres de Sanidade. São Paulo, 56p. ASEA, G.; G. BIGIRWA; E. ADILAPA; S.A.P. OWERA; R.C. PRATT; P.E. LIPPS, 2002. Effect of Cercospora zeae-maydis Infested Maize Residue on Progress and Spread of Gray Leaf Spot of Maize in Central Uganda. Ann. App. Biol., 140:177-185. BRANDÃO, A.M.; F.C. JULIATTI; C.H. BRITO; L.S. GOMES, 2001. Manejo Integrado de Cercosporiose (Cercospora zeae-maydis) em Milho, no Cerrado Brasileiro. Fitopatol. Bras., 26:401-402.

CARSON, M.L.; M.M. GOODMAN; S.M. WILLIAMSON, 2002.

Variation in Aggressiveness among Isolates of Cercospora from Maize as a Potential Cause of Genotype-Environment Interaction in Gray Leaf Spot Trials. Plant Disease, 86:1089-1093.

DONAHUE, P.J.; E.L. STROMBERG; S.L. MYERS, 1991. Inheritance of Reaction to Gray Leaf Spot in a Diallel Cross of 14 Maize Imbreds. Crop Science, 41:925-931.

DUNKLE, L.D., M.L. CARSON, 1998. Genetic Variation in Cercospora and the Impact on Selecting for Resistance to Gray Leaf Spot of Corn. In: ANNUAL CORN \& SORGHUM RESEARCH CONFERENCE, 53., Chicago, 1998. Proceedings. Washington: American Seed Trade Association. p.334-347.

FANTIN, G.M.; K.R. BRUNELLI; I.C. RESENDE; A.P. DUARTE, 2001. A Mancha de Cercospora do Milho. Campinas: IAC, 19p. (Boletim Técnico, 192)

FERNANDES, F.T.; E. OLIVEIRA, 2000. A Mancha por Cercospora em Milho. Sete Lagoas: Embrapa, 1p. (Comunicado Técnico, 16) GEVERS, H.O.; J.K. LAKE; T. HOHLS, 1994. Diallel Crossanalysis of Resistance to Gray Leaf Spot in Maize. Plant Disease, 78:379383.

LATTERELL, F.M.; A.E. ROSSI, 1983. Gray Leaf Spot of Corn: A

Disease on the Move. Plant Disease, 67:842-847.

LIPPS, P.E.; D.G. WHITE; J.E. AYERS; L.D. DUNKLE, 1998. Gray Leaf Spot: a Global Threat to Corn Production. NCR-25 Tecnical Committee on Corn and Sorghum Diseases. APSNet Feature: Americam Phytopathological Society. Publicação on line. 4p. 
MUNKVOLD, G.P.; C.A. MARTINSON; J.M. SHRIVER; P.M. DIXON, 2001. Probabilities for Profitable Fungicide Use Againt Gray Leaf Spot in Hybrid Maize. Phytopathology, 91:477-484.

PINTO, N.F J.A.; B. ANGELIS; M.H. HABE, 2002. Avaliação da Eficiência de Fungicidas no Controle da Cercosporiose (Cercospora zeae-maydis) na Cultura do Milho. In: CONGRESSO NACIONAL DE MILHO E SORGO, 24, Florianópolis, 2002. Meio Ambiente e a Nova Agenda para o Agronegócio de Milho e Sorgo: Resumos. Sete Lagoas: ABMS/Embrapa Milho e Sorgo/Epagri, p.117. RAMOS, C.R.B.A.; C.C.E. MENEZES; M.A.B. MORANDI, 2001 a. Avaliação de Fungicidas para Controle da Cercosporiose do Milho. Fitopatol. Brasil., 26:322. RAMOS, C.R.B.A.; C.C.E. MENEZES; M.A.B. MORANDI, $2001 \mathrm{~b}$. Controle Químico da Cercosporiose do Milho. Fitopatol. Brasil., 26:322.

SAGHAI MAROOF, M.A.; S.W. VAN SCOYOC; Y.G. YU; E.L. STROMBERG, 1993. Gray Leaf Spot Disease of Maize: Rating Methodology and Inbred Line Evaluation. Plant Disease, 77:583587.

WARD, J.M.J.; T. HOHLS; M.D. LAING; F.H.J. RIJKENBERG, 1996. Fungicides Responses of Maize Inbreds to Gray Leaf Spot. Eur. J. Plant Pathol., 102:765-771.

WARD, J.M.J.; M.D. LAING; D.C. NOWELL, 1997a. Chemical Control of Maize Gray Leaf Spot. Crop Protection, 16:265-271. WARD, J.M.J.; M.D. LAING; F.H.J. RIJKENBERG, 1997b. Frequency and Timing of Fungicide Applications for the Control of Gray Leaf Spot in Maize. Plant Disease, 81:41-48. WARD, J.M.J.; E.L. STROMBERG; D.C. NOWELL; F.W. NUTTER JR., 1999. Gray Leaf Spot: a Disease of Global Importance in Maize Production. Plant Disease, 83:884-895. WHITE, D.G. (Ed.) 2000. Compendium of Corn Diseases. 3.ed. St.

Paul: American Phytopathological Society, 78p. 